\title{
Discovery of New a-Glucosidase Inhibitors: Structure-Based Virtual Screening and Biological Evaluation
}

\begin{abstract}
Shan-Kui Liu ${ }^{1}$, Haifang Hao ${ }^{1}$, Yuan Bian ${ }^{1}$, Yong-Xi Ge ${ }^{1}$, Shengyuan Lu ${ }^{1}$, Hong-Xu Xie ${ }^{1}$, Kai-Ming Wang ${ }^{1}$, Hongrui Tao $^{2}$, Chao Yuan ${ }^{3}$, Juan Zhang ${ }^{1 *}$, Jie Zhang ${ }^{4}$, Cheng-Shi Jiang ${ }^{1 *}$ and Kongkai Zhu ${ }^{1,5 *}$

${ }^{1}$ School of Biological Science and Technology, University of Jinan, Jinan, China, ${ }^{2}$ Drug Discovery and Design Center, State Key Laboratory of Drug Research, Shanghai Institute of Materia Medica, Chinese Academy of Sciences, Shanghai, China, ${ }^{3}$ Zoucheng Administration for Market Regulation, Zoucheng, China, ${ }^{4}$ Lunan Pharmaceutical Group Corporation, Linyi, China, ${ }^{5}$ Shandong Key Laboratory of Biochemical Analysis, College of Chemistry and Molecular Engineering, Qingdao University of Science and Technology, Qingdao, China
\end{abstract}

OPEN ACCESS

Edited by:

Zhongjie Liang,

Soochow University, China

Reviewed by:

Xuben Hou,

Shandong University, China

Xiao Jun Yao,

Macau University of Science and

Technology, Macau

${ }^{*}$ Correspondence:

Juan Zhang

bio_zhangj@ujn.edu.cn

Cheng-Shi Jiang

bio_jiangcs@ujn.edu.cn

Kongkai Zhu

bio_zhukk@ujn.edu.cn

Specialty section:

This article was submitted to

Medicinal and Pharmaceutical

Chemistry,

a section of the journal

Frontiers in Chemistry

Received: 09 December 2020

Accepted: 20 January 2021

Published: 08 March 2021

Citation:

Liu S-K, Hao H, Bian Y, Ge Y-X, Lu S, Xie $H-X$, Wang $K-M$, Tao $H$, Yuan $C$,

Zhang J, Zhang J, Jiang C-S and

Zhu K (2021) Discovery of New

$\alpha$-Glucosidase Inhibitors: Structure-

Based Virtual Screening and

Biological Evaluation.

Front. Chem. 9:639279.

doi: $10.3389 /$ fchem.2021.639279
a-Glycosidase inhibitors could inhibit the digestion of carbohydrates into glucose and promote glucose conversion, which have been used for the treatment of type 2 diabetes. In the present study, 52 candidates of a-glycosidase inhibitors were selected from commercial Specs compound library based on molecular docking-based virtual screening. Four different scaffold compounds $(7,22,37$, and 44) were identified as a-glycosidase inhibitors with $I_{50}$ values ranging from 9.99 to $35.19 \mu \mathrm{M}$. All these four compounds exerted better inhibitory activities than the positive control (1deoxynojirimycin, $\left.I_{50}=52.02 \mu \mathrm{M}\right)$. The fluorescence quenching study and kinetic analysis revealed that all these compounds directly bind to a-glycosidase and belonged to the noncompetitive a-glycosidase inhibitors. Then, the binding modes of these four compounds were carefully investigated. Significantly, these four compounds showed nontoxicity $\left(\mathrm{IC}_{50}>100 \mu \mathrm{M}\right)$ toward the human normal hepatocyte cell line (LO2), which indicated the potential of developing into novel candidates for type 2 diabetes treatment.

Keywords: $\alpha$-glycosidase, virtual screening, cytotoxicity, type 2 diabetes, molecular docking

\section{INTRODUCTION}

Diabetes is a metabolic disorder that causes high blood sugar and could directly increase the risk of other deadly diseases, such as cancer, stroke, and cardiovascular diseases (Cohen and Goedert, 2004; Zeng et al., 2019). According to the statistics of the World Health Organization (WHO), about 422 million people suffered from diabetes in 2014 around the world, and its prevalence is projected to be 642 million by 2040 (Reusch and Manson, 2017; World Health Organization, 2020). The ineffective use of insulin could result in the type 2 diabetes and accounts for more than $90 \%$ of diabetes cases (Proença et al., 2017).

Controlling blood glucose levels is thought to be the main strategy for treating diabetes and reducing diabetes complications (Ye et al., 2019). a-Glucosidase is a key carbohydrate hydrolase that regulates blood glucose by specifically hydrolyzing 1,4- $\alpha$-glucopyranosidic bond to produce a-glucose (Kazmi et al., 2018). Early studies have shown that the inhibition of a-glucosidase activity could retard the absorption of glucose and decrease the postprandial blood glucose levels 


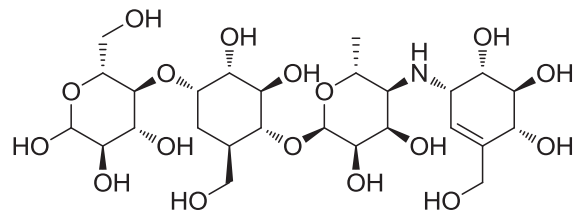

Acarbose<smiles>OCC1=C[C@@H](NC(CO)CO)[C@H](O)C(O)C1O</smiles>

Voglibose

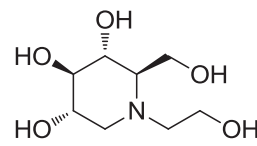

Miglitol

FIGURE 1 | Clinically Approved $\alpha$-glucosidase inhibitors.

(Park et al., 2008; Kim et al., 2019). Therefore, a-glucosidase has been taken as a key target for treating diabetes, and the inhibitors of a-glucosidase can be developed into effective therapeutic drugs to treat this disease (Li et al., 2010). $\alpha$-Glucosidase inhibitors such as acarbose, miglitol, and voglibose (shown in Figure 1) are the most well-known ones (Joshi et al., 2015). Acarbose, the first approved drug in a-glucosidase inhibitor category, was used to delay the release of glucose from polysaccharides by binding with a-glucosidase. Voglibose was used to discontinue the uptake and hydrolysis of saccharides by selectively inhibiting a-glucosidase vs. pancreatic a-amylase and lactase. Miglitol, the first pseudomonosaccharide $a$-glucosidase inhibitor, was approved to reduce postprandial glucose (Hossain et al., 2020). However, some unexpected adverse effects (for instance, flatulence, diarrhea, and stomachache) limited their clinical application. Based on this background, numerous efforts have been carried out to discover new a-glucosidase inhibitors from diverse sources, such as natural products and chemical synthetic compounds (Chen et al., 2017; Liu and Ma, 2017; Abbas et al., 2019; Dhameja and Gupta, 2019).

Virtual screening has been proven to be a very effective tool capable of providing drug hits or leads with structural diversity and makes drug discovery faster and more efficient (Kitchen et al., 2004; Kontoyianni, 2017). In this study, molecular docking-based virtual screening on Specs database was conducted to identify a-glucosidase inhibitors with new chemotypes. After testing the purchased 52 compounds that were obtained by docking screening, four compounds, namely, 7, 22, 37, and 44 with different scaffolds, were disclosed as new a-glycosidase inhibitors. Kinetic analysis of these compounds revealed that they inhibited a-glycosidase activity in a noncompetitive type. Then, the binding modes of these compounds with a-glycosidase were investigated, and the results indicated that all of these compounds could be well located in the acarbose-binding site and displayed very similar binding poses. Moreover, the cytotoxicity of these compounds toward the human normal hepatocyte cell line (LO2) was evaluated. The present results provided new a-glycosidase inhibitors serving as hit compounds for developing novel medications used in the treatment of type 2 diabetes.

\section{METHODS AND MATERIALS}

\section{Molecular Docking-Based Virtual Screening}

The protein coordinates in the a-glycosidase crystal complex structure (PDB code 3W37) were prepared by the Protein
Preparation Wizard panel inserted in the Maestro with the default settings. Residues within $15 \AA$ centered on acarbose were defined as compound-binding sites in which the docking grid was generated by the Receptor Grid Generation panel. The default settings were adopted for the cutoff, neutralization, etc. The docked compounds in Specs database were prepared with LigPrep panel. Then, the prepared compounds were docked to the aforementioned docking gird with extra precision (XP) mode. "Clustering Molecules" protocol inserted in Pipeline Pilot 7.5 was employed to achieve the cluster analysis. The top ranked compounds assessed by XP GScore were clustered into 30 clusters. To increase the diversity of selected compounds, at least one candidate was selected in each cluster. In addition, we gave priority to the compounds with simple structure and/or small molecular weight.

\section{a-Glycosidase Inhibitory Assay}

The a-glucosidase inhibitory evaluation of the purchased 52 compounds was performed according to the previously described protocol (Tang et al., 2014; Ye et al., 2019). a-Glucosidase (Sigma, G5003) derived from baker's yeast, and pNPG (Sigma, N1377) and the substrate were both purchased from Sigma-Aldrich. 1-Deoxynojirimycin was used as the positive control. The tested compounds and 1deoxynojirimycin were dissolved in DMSO, the a-glucosidase and the substrate pNPG were both dissolved in phosphate buffer $(\mathrm{pH}=6.8)$. The compounds and $\alpha$-glucosidase were preincubated in phosphate buffer $\left(37^{\circ} \mathrm{C}, 15 \mathrm{~min}\right)$. Then, $25 \mu \mathrm{L}$ substrate buffer was added to the system to start the reaction, and the incubation was continued at $37^{\circ} \mathrm{C}$ for $15 \mathrm{~min}$. Finally, the reaction was terminated by the addition of $50 \mu \mathrm{L} \quad 0.2 \mathrm{M}$ reaction termination solution. The optical density (OD) was measured at an absorbance wavelength of $405 \mathrm{~nm}$ using a microplate reader (Tecan, Switzerland). The $\mathrm{IC}_{50}$ values were estimated with six different concentrations, and each sample was measured three times in parallel experiments.

\section{Fluorescence Quenching Experiment}

According to the previously reported method (AguilarMoncayo et al., 2010), all fluorescence spectra were measured on a fluorescence spectrophotometer (Agilent Cary Eclipse) equipped with a $10.0-\mathrm{mm}$ quartz cell and a thermostat bath. In the fluorescence spectrophotometer, a-glucosidase $(1 \mathrm{U} / \mathrm{ml})$ was pretreated with certain concentrations of inhibitors for $30 \mathrm{~min}$ at $37^{\circ} \mathrm{C} .100 \mu \mathrm{L}$ of the above solution 

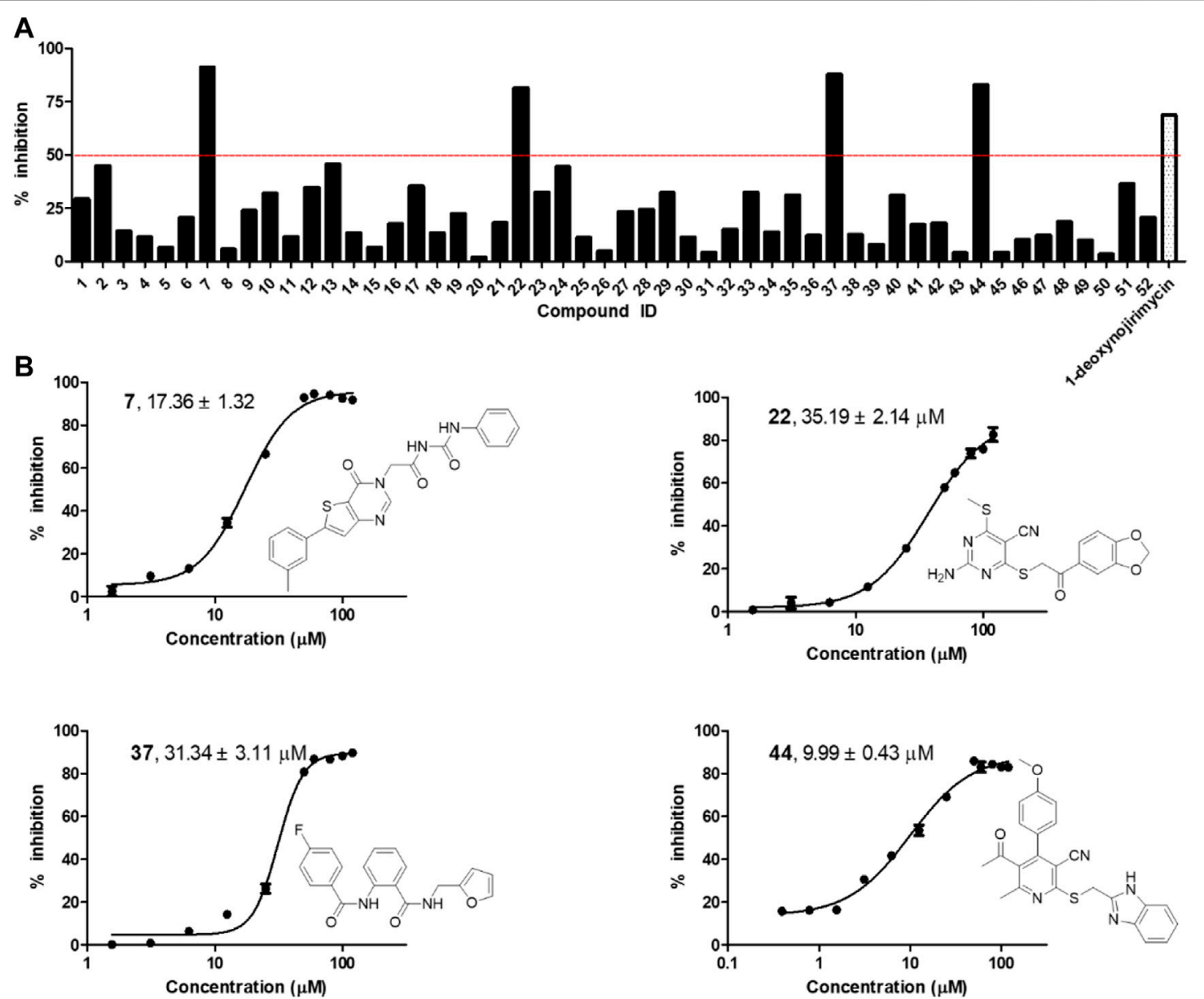

FIGURE 2 | (A) $\alpha$-glycosidase inhibitory activity of the 52 selected candidates at $100 \mu \mathrm{M}$; (B) The chemical structures and IC ${ }_{50}$ curves of compounds 7, 22, 37, and 44. $I C_{50}$ data are shown as mean $\pm S D$ of three independent experiments.

(pH 6.8) was added accurately to the quartz cell. The blank was used for buffer spectrum values. The fluorescence emission spectra were measured at $37^{\circ} \mathrm{C}$. The excitation wavelength was $290 \mathrm{~nm}$, and the emission spectrum was recorded from 320 to $500 \mathrm{~nm}$.

\section{Kinetic Assay}

The inhibition type of the inhibitors against a-glucosidase activities was evaluated based on a described method (Hou et al., 2009). Increasing concentrations of substrates pNPG were used in the absence or presence of tested compounds at four different concentrations around the $\mathrm{IC}_{50}$ values. The inhibitory kinetics of the investigated compounds on a-glucosidase was analyzed using the Lineweaver-Burk plot of the substrate concentration and velocity.

\section{Cell Viability Assay}

The LO2 cell line was cultured in a proper medium supplemented with $10 \%$ fetal bovine serum in a humidified atmosphere of $5 \%$ $\mathrm{CO}_{2}$ at $37^{\circ} \mathrm{C}$. Cell suspensions were plated in 96-well plates at a density of $2 \times 10^{4}$ cells $/ \mathrm{cm}^{3}$. Compounds were solubilized in DMSO at six different concentrations. After incubation for $24 \mathrm{~h}$, the cells were treated with various concentrations of tested substances for $48 \mathrm{~h}$ and then incubated with $100 \mu \mathrm{L}$ of MTT at $37^{\circ} \mathrm{C}$ for $2 \mathrm{~h}$. The formazan dye product was measured by the absorbance at $490 \mathrm{~nm}$ on a Tecan Spark multimode microplate reader (Switzerland).

\section{RESULTS AND DISCUSSIONS}

\section{Fifty-Two Candidates of $\alpha$-Glycosidase} Inhibitor Were Selected From the Molecular Docking-Based Virtual Screening Result

As the crystal structure of a-glycosidase-acarbose complex has been determined (PDB code 3W37) (Tagami et al., 2013), molecular docking-based virtual screening could be performed. Specs database that contains 200,000 compounds was chosen as the screening database. The redock result of 

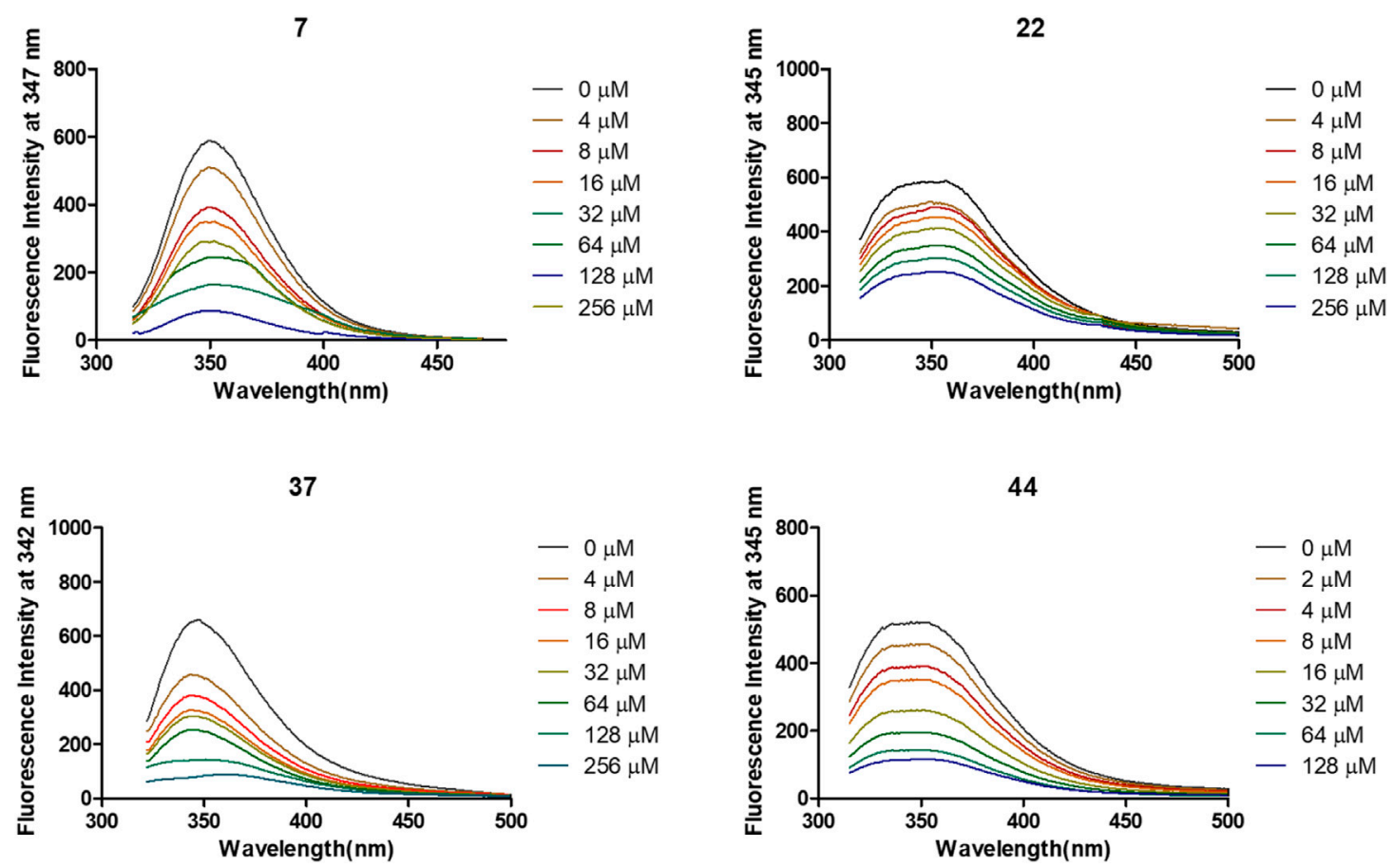

FIGURE 3 | Variation of fluorescence emission spectra of a-glycosidase $(1 \mathrm{U} / \mathrm{ml})$ in the presence of compounds $7,22,37$, and 44 with increasing concentration for $30 \mathrm{~min}$ at $37^{\circ} \mathrm{C}$

7

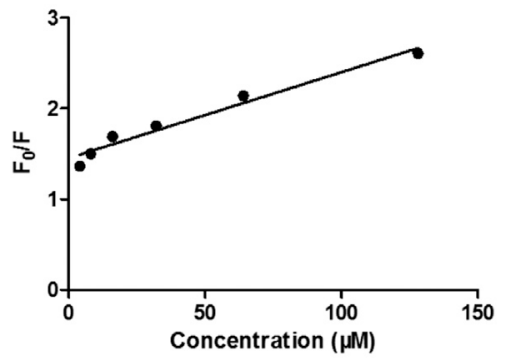

37

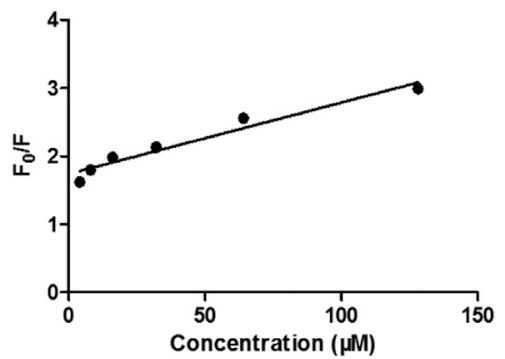

22

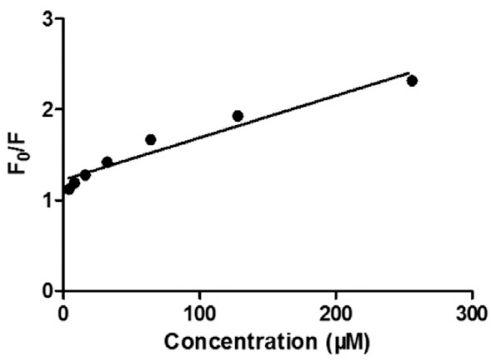

44

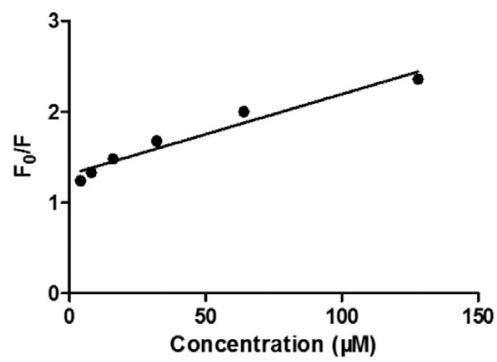

FIGURE 4 | Stern-Volmer plots for the fluorescence quenching of $\alpha$-glycosidase by compounds 7, 22, 37, and 44.

acarbose (Supplementary Figure S1) declared that GLIDE program (Halgren et al., 2004) inserted in the Schrödinger program suite could well reproduced the binding mode of acarbose in the crystal structure. The top 300 molecules ranked by the docking score were selected for the following cluster analysis. Finally, 52 compounds were retained and 

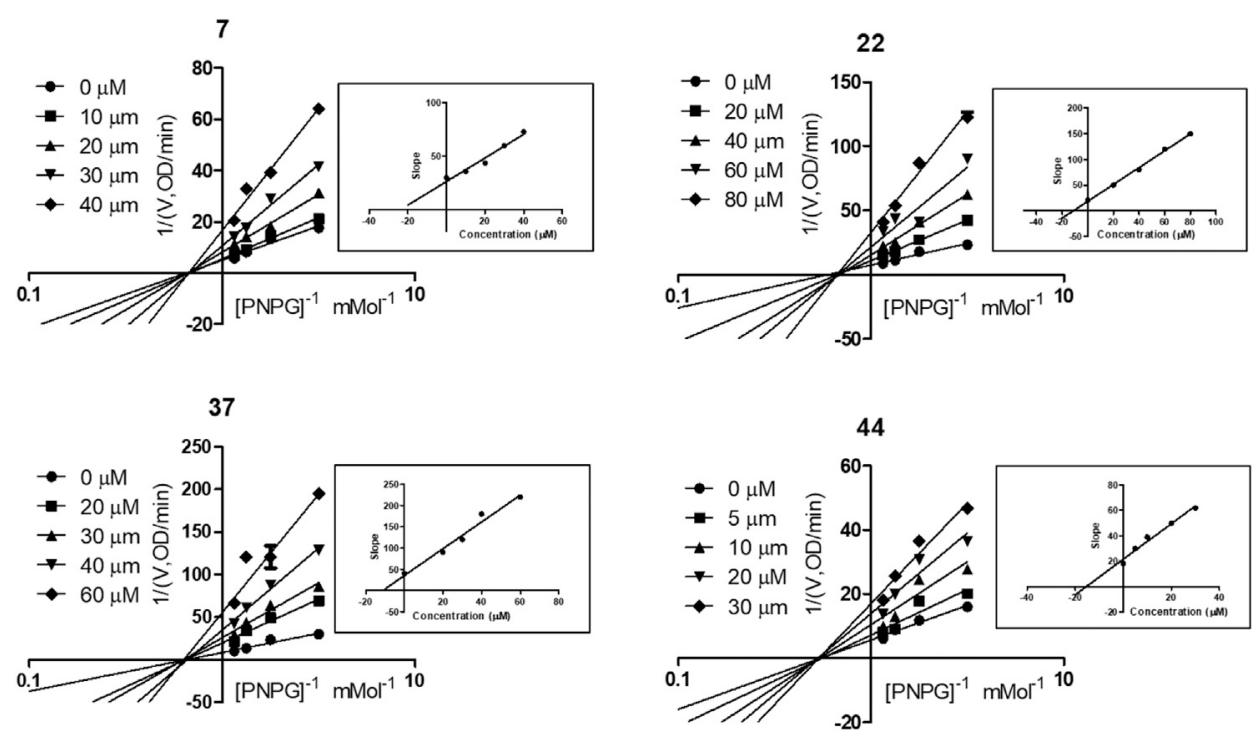

FIGURE 5 | Kinetic assay on a-glycosidase inhibition by compounds 7, 22, 37, and 44, respectively. Lineweaver-Burk reciprocal plots of initial velocity and increasing substrate (PNPG) concentration with secondary plot of slopes vs. the concentration of compounds.
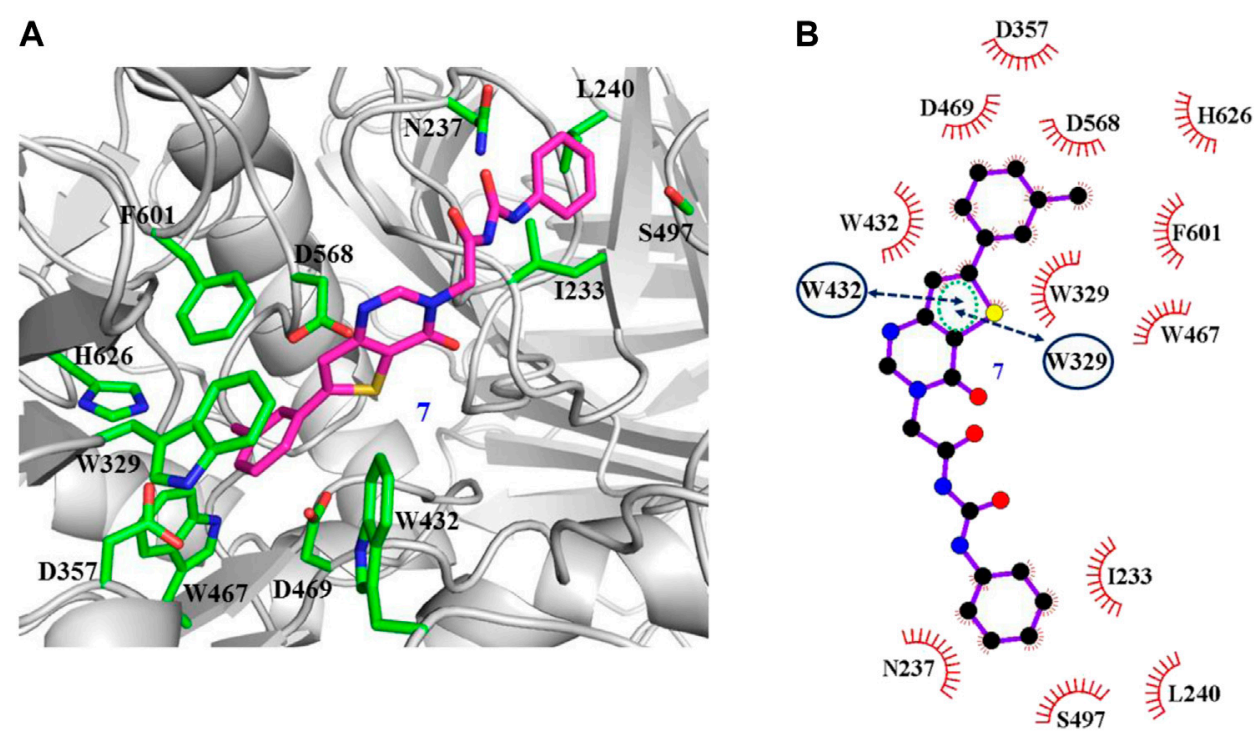

FIGURE 6 | Docking pose of compound 7 bound to the acarbose binding site in a-glycosidase. (A) The three-dimensional interacting modes between 7 and $\alpha$-glycosidase. $\alpha$-Glycosidase, 7 and the interacting residues were shown as cartoon, sticks (carbon atoms colored in magenta), sticks (carbon atom colored in green), respectively. (B) Schematic representation displayed the hydrophobic interactions (shown as starbursts) and Pi-Pi interactions (shown as oval) of 7 with a-Glycosidase.

purchased from the Specs database supplier for further a-glycosidase enzymatic inhibition activity evaluation.

\section{In vitro Inhibition Test Against a-Glycosidase Identified Four Active Compounds 7, 22, 37, and 44}

The selected 52 candidates were initially evaluated for their inhibitory ratios against $\alpha$-glycosidase at $100 \mu \mathrm{M}$ with 1 - deoxynojirimycin as positive reference. The a-glycosidase enzymatic inhibition bioassay results indicated that four compounds, namely, 7, 22, 37, and 44 with representing totally different scaffolds, exhibited an inhibition ratio above $50 \%$ at $100 \mu \mathrm{M}$ (Figure $2 \mathrm{~A}$ ). Then, the $\mathrm{IC}_{50}$ values of these four compounds were further determined. As shown in Figure 2B, compounds 7, 22, 37, and 44 displayed IC $_{50}$ values of $17.36 \pm 1.32,35.19 \pm 2.14,31.34 \pm 3.11$, and $9.99 \pm 0.43 \mu \mathrm{M}$, respectively. All of them showed better activity than the 

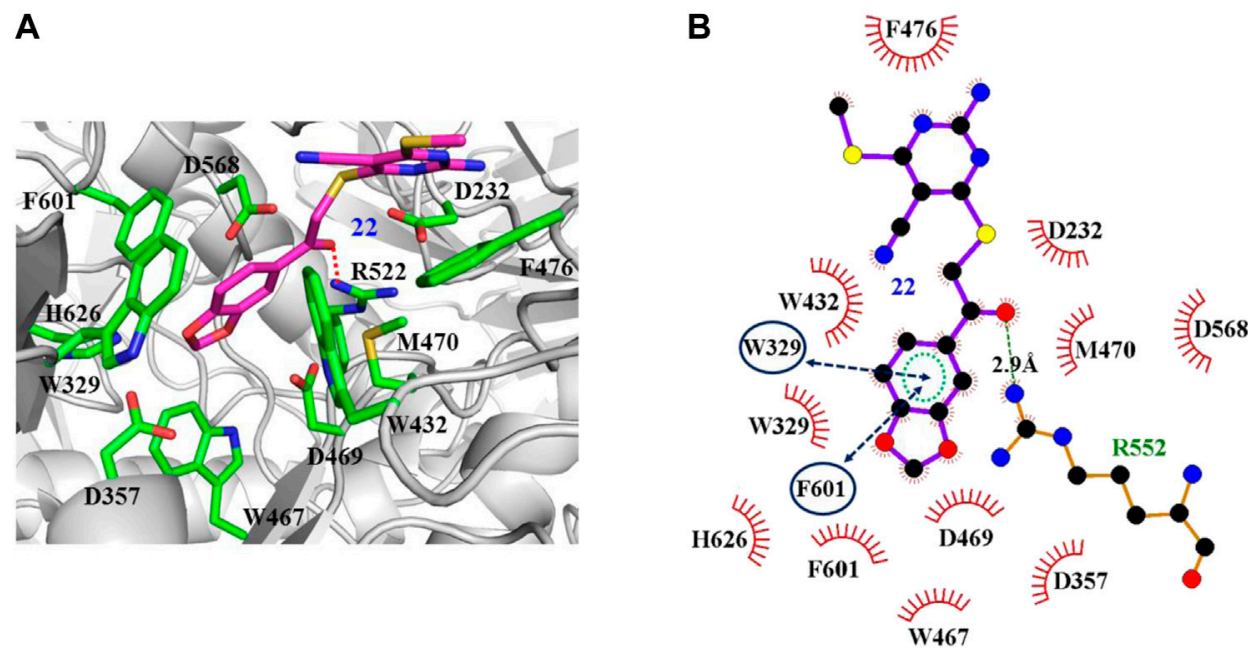

FIGURE 7 | Docking pose of compound 22 bound to the acarbose binding site in a-glycosidase. (A) The three-dimensional interacting modes between 22 and a-glycosidase. $\alpha$-Glycosidase, 22 and the interacting residues were shown as cartoon, sticks (carbon atoms colored in magenta), sticks (carbon atom colored in green), respectively. (B) Schematic representation displayed the hydrophobic interactions (shown as starbursts), Pi-Pi interactions (shown as oval), and $\mathrm{H}$-bond interactions (denoted by dotted green lines) of 22 with $\alpha$-Glycosidase.
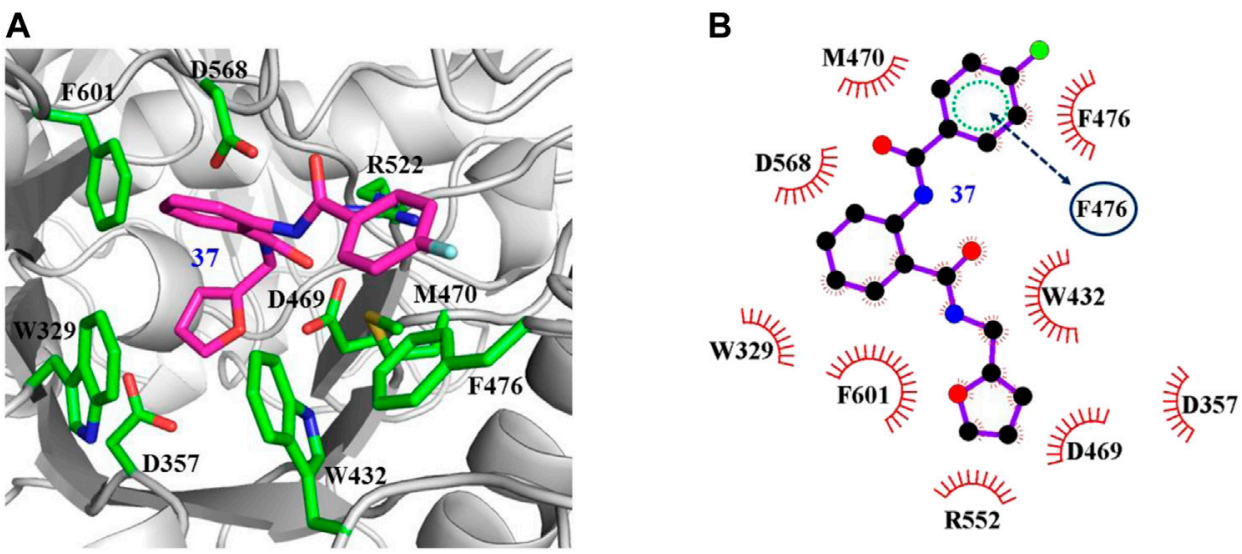

FIGURE 8 | Docking pose of compound 37 bound to the acarbose binding site in a-glycosidase. (A) The three-dimensional interacting modes between 37 and a-glycosidase. $\alpha$-Glycosidase, 37 and the interacting residues were shown as cartoon, sticks (carbon atoms colored in magenta), sticks (carbon atom colored in green), respectively. (B) Schematic representation displayed the hydrophobic interactions (shown as starbursts) of 37 with a-Glycosidase.

positive reference control 1-deoxynojirimycin $\left(\mathrm{IC}_{50}, 52.02 \pm\right.$ $3.78 \mu \mathrm{M})$, and compound 44 exhibited the most potent activity.

\section{Fluorescence Quenching Assay Confirmed the Binding of $7,22,37$, and 44 to a-Glycosidase}

The interactions of $7,22,37$, and 44 with a-glycosidase were explored through the fluorescence quenching experiments. As displayed in Figure 3, the variations of the intrinsic fluorescence emission of $\alpha$-glycosidase $(2 \mu \mathrm{M})$ in the presence of increasing concentration of molecules $7,22,37$, and 44, respectively, were recorded at $37^{\circ} \mathrm{C}$ with the wavelength range from 320 to $500 \mathrm{~nm}$.
The intrinsic fluorescence emission peak at $345 \mathrm{~nm}$ was observed after being excited at $290 \mathrm{~nm}$.

After treated by compounds 7, 22, 37, and 44 with increasing concentration (Figure 4), the fluorescence intensities of enzyme in all tested systems were gradually quenched in a type of concentration-dependent manner. Thus, these results confirmed the binding of these inhibitors to a-glycosidase.

\section{Kinetic Study on $\alpha$-Glycosidase Inhibition Declared the Noncompetitive Manner of These Four Compounds}

To explore the mechanism of the interaction modes of compounds $7,22,37$, and 44 with the enzyme, kinetic assay 
A

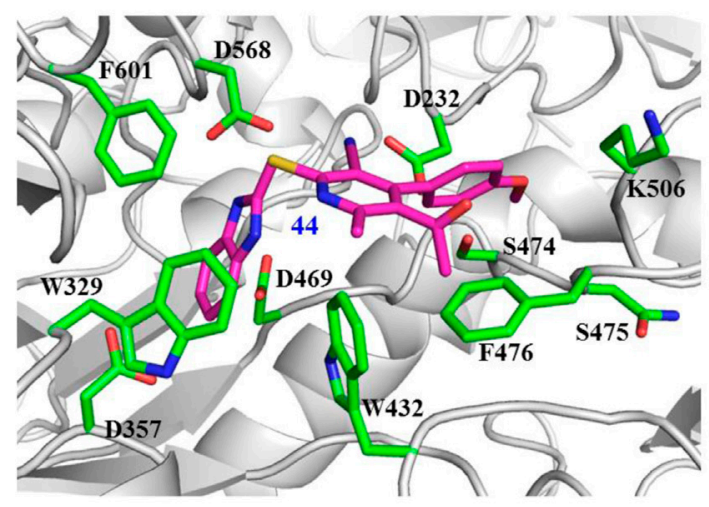

B

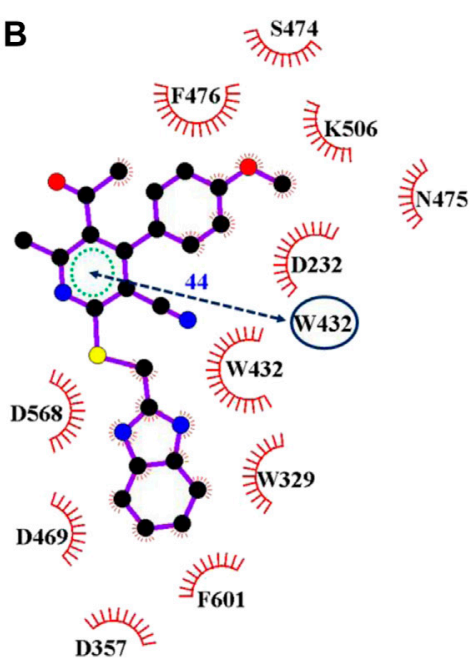

FIGURE 9 |Docking pose of compound 44 bound to the acarbose binding site in a-glycosidase. (A) The three-dimensional interacting modes between 44 and a-glycosidase. Compound 44 and the interacting residues were shown as cartoon, sticks (carbon atoms colored in magenta), sticks (carbon atom colored in green), respectively. (B) Schematic representation displayed the hydrophobic interactions (shown as starbursts) and $\mathrm{Pi}-\mathrm{Pi}$ interactions (shown as oval) of 44 with a-Glycosidase.

was conducted to study their inhibition types using Lineweaver-Burk plot analysis (Wang et al., 2004; Sun et al., 2018). The results shown in Figure 5 indicate that compounds 7 , 22,37 , and 44 were noncompetitive a-glycosidase inhibitors, with estimated $K_{\mathrm{i}}$ values of $24.18,11.34,11.27$, and $15.39 \mu \mathrm{M}$, respectively.

\section{Molecular Docking Simulation Revealed the Binding Mode of These Four Compounds}

The interaction mechanisms of compounds 7, 22, 37, and 44 with a-glycosidase were carefully analyzed with the molecular docking results, as shown in Figures 6-9. All these four compounds could well bind to the allosteric sites away from the active site (Asp214, Glu276, and Asp349) (Ye et al., 2019) in a-glycosidase and formed hydrophobic interactions with nearby residues. These results were consistent with the noncompetitive property. The docking scores of these four hits were $-3.811,-2.825,-3.627$, and -6.283. Specifically, inhibitor 7 established hydrophobic interactions with residues D357, D469, W432, N237, S497, L240, I233, W329, W467, F601, H626, and D568 and formed $\mathrm{Pi}-\mathrm{Pi}$ stacking with residues W329 and W432. Compound 22 formed hydrophobic interactions with residues W329, F476, D357, D469, W432, D232, M470, W467, F601, H626, and D568 and formed Pi-Pi stacking with residues W329 and F601. Besides, 22 established $\mathrm{H}$ bond interaction with $\mathrm{R} 552$ residue. Compound 37 formed hydrophobic interaction with residues M470, W329, F476, D357, D469, W432, F601, D568, and $\mathrm{R} 552$ and formed $\mathrm{Pi}-\mathrm{Pi}$ stacking with residue F476. Additionally, compound 44 formed hydrophobic interaction with residues S474, W329, F476, D357, D469, W432, N475, D568, D232, F601, and K506 and formed Pi-Pi stacking with residue W432. From these data, we could find that residues
W432, W329, F601, D357, D469, and D568 were the key residues contributing interaction with all of the four compounds.

\section{In vitro Cytotoxicity}

Since most of the drugs are metabolized in the liver, there is a great focus on the hepatic safety of new medicines. Thus, the cytotoxicity of inhibitors 7, 22, 37, and 44 was evaluated in human normal hepatocyte (LO2) cells using the MTT method (Ge et al., 2020). The results disclosed that all of these compounds had $\mathrm{IC}_{50}$ values more than $100 \mu \mathrm{M}$ toward LO2 cells, suggesting they are nontoxic toward liver cells. Thus, further structural optimization and biological evaluation for $7,22,37$, and 44 deserved further investigation.

\section{CONCLUSION}

In this study, four novel a-glycosidase inhibitors 7, 22, 37, and 44 with distinct structural features were identified through virtual screening and in vitro evaluation. Among them, compound 44 had the best $\alpha$-glycosidase inhibitory activity with $\mathrm{IC}_{50}$ and $K_{\mathrm{i}}$ values of $9.99 \pm 0.43$ and $15.39 \mu \mathrm{M}$, respectively. The fluorescence quenching experiment indicated all these compounds could directly bind to a-glycosidase, and the kinetic study revealed a noncompetitive a-glycosidase inhibitory mechanism of these compounds toward a-glycosidase. In addition, binding mode analysis provided the detailed binding mechanism of these four a-glycosidase inhibitors, which made further structural optimization feasible. Moreover, the in vitro cytotoxicity bioassay demonstrated these a-glycosidase inhibitors were nontoxic toward LO2 cells. Based on these results, these compounds can serve as promising hit compounds for further bioactivity optimization and anti-type 2 diabetes study. 


\section{DATA AVAILABILITY STATEMENT}

The original contributions presented in the study are included in the article/Supplementary Material, further inquiries can be directed to the corresponding authors.

\section{AUTHOR CONTRIBUTIONS}

All authors made significant contributions to the preparation of the manuscript and approved it before submission. KZ, C-SJ, and $\mathrm{JuZ}$ developed the paper design and finalized the manuscript. $\mathrm{S}-\mathrm{KL}$ wrote the manuscript; $\mathrm{HH}$ and $\mathrm{YB}$ performed molecular docking study; Y-XG, SL, and H-XX helped to collect the data and complete the figure; K-MW and HT edited the references and revised the style of the manuscript, $\mathrm{CY}$ and JiZ revised the manuscript.

\section{REFERENCES}

Abbas, G., Al-Harrasi, A., Hussain, H., Hamaed, A., and Supuran, C. T. (2019). The management of diabetes mellitus-imperative role of natural products against dipeptidyl peptidase-4, a-glucosidase and sodium-dependent glucose Cotransporter 2 (SGLT2). Bioorg. Chem. 86, 305-315. doi:10.1016/j.bioorg. 2019.02.009

Aguilar-Moncayo, M., García-Moreno, M. I., Stütz, A. E., García, Fernández, J. M., Wrodnigg, T. M., and Ortiz-Mellet, C. (2010). Fluorescent-tagged $\mathrm{sp}^{2}$ iminosugars with potent $\beta$-glucosidase inhibitory activity. Bioorg. Med. Chem. 18 (21), 7439-7445. doi:10.1016/j.bmc.2010.09.003

Chen, H., Xiong, L., and Wang, W. J. (2017). Reviews on a-glucosidase inhibitor from plant secondary metabolites. Zhongguo Zhong Yao Za Zhi 42 (15), 2915-2924. doi:10.19540/j.cnki.cjcmm.20170714.021

Cohen, P., and Goedert, M. (2004). GSK3 inhibitors: development and therapeutic potential. Nat. Rev. Drug Discov. 3 (6), 479-487. doi:10.1038/nrd1415

Dhameja, M., and Gupta, P. (2019). Synthetic heterocyclic candidates as promising a-glucosidase inhibitors: an overview. Eur. J. Med. Chem. 176, 343-377. doi:10. 1016/j.ejmech.2019.04.025

Ge, Y. X., Cheng, Z. Q., Zhou, L., Xie, H. X., Wang, Y. Y., Zhu, K., et al. (2020). Synthesis and biological evaluation of quinoline/cinnamic acid hybrids as amyloid-beta aggregation inhibitors. Monatsh Chem. 151, 845-852. doi:10. 1007/s00706-020-02609-2

Halgren, T. A., Murphy, R. B., Friesner, R. A., Beard, H. S., Frye, L. L., Pollard, W. T., et al. (2004). Glide: a new approach for rapid, accurate docking and scoring. 2. Enrichment factors in database screening. J. Med. Chem. 47 (7), 1750-1759. doi:10.1021/jm030644s

Hossain, U., Das, A. K., Ghosh, S., and Sil, P. C. (2020). An overview on the role of bioactive a-glucosidase inhibitors in ameliorating diabetic complications. Food Chem. Toxicol. 145, 111738. doi:10.1016/j.fct.2020.111738

Hou, W., Li, Y., Zhang, Q., Wei, X., Peng, A., Chen, L., et al. (2009). Triterpene acids isolated from Lagerstroemia speciosa leaves as alpha-glucosidase inhibitors. Phytother Res. 23 (5), 614-618. doi:10.1002/ptr.2661

Joshi, S. R., Standl, E., Tong, N., Shah, P., Kalra, S., and Rathod, R. (2015). Therapeutic potential of $\alpha$-glucosidase inhibitors in type 2 diabetes mellitus: an evidence-based review. Expert Opin. Pharmacother. 16 (13), 1959-1981. doi:10. 1517/14656566.2015.1070827

Kazmi, M., Zaib, S., Ibrar, A., Amjad, S. T., Shafique, Z., Mehsud, S., et al. (2018). A new entry into the portfolio of a-glucosidase inhibitors as potent therapeutics for type 2 diabetes: Design, bioevaluation and one-pot multi-component

\section{FUNDING}

This research work was financially supported by the Major Science and Technology Innovation Project of Shandong Province (No. 2019JZZY011116), National Natural Science Foundation of China (Nos. 21672082 and 81803438), Natural Science Foundation of Shandong Province (Nos. ZR2019YQ31 and ZR201910300056), Key Technology Research and Development Program of Shandong (No. 2019GSF108043), and Science and Technology Project of University of Jinan (No. XKY2004).

\section{SUPPLEMENTARY MATERIAL}

The Supplementary Material for this article can be found online at: https://www.frontiersin.org/articles/10.3389/fchem.2021.639279/ full\#supplementary-material.

synthesis of diamine-bridged coumarinyl oxadiazole conjugates. Bioorg. Chem. 77, 190-202. doi:10.1016/j.bioorg.2017.12.022

Kim, J. H., Kim, H. Y., and Jin, C. H. (2019). Mechanistic investigation of anthocyanidin derivatives as a-glucosidase inhibitors. Bioorg. Chem. 87, 803-809. doi:10.1016/j.bioorg.2019.01.033

Kitchen, D. B., Decornez, H., Furr, J. R., and Bajorath, J. (2004). Docking and scoring in virtual screening for drug discovery: methods and applications. Nat. Rev. Drug Discov. 3 (11), 935-949. doi:10.1038/nrd1549

Kontoyianni, M. (2017). Docking and virtual screening in drug discovery. Methods Mol. Biol. 1647, 255-266. doi:10.1007/978-1-4939-7201-2_18

Li, D. Q., Qian, Z. M., and Li, S. P. (2010). Inhibition of three selected beverage extracts on alpha-glucosidase and rapid identification of their active compounds using HPLC-DAD-MS/MS and biochemical detection. J. Agric. Food Chem. 58 (11), 6608-6613. doi:10.1021/jf100853c

Liu, Z., and Ma, S. (2017). Recent advances in synthetic $\alpha$-glucosidase inhibitors. ChemMedChem 12 (11), 819-829. doi:10.1002/cmdc.201700216

Park, H., Hwang, K. Y., Oh, K. H., Kim, Y. H., Lee, J. Y., and Kim, K. (2008). Discovery of novel alpha-glucosidase inhibitors based on the virtual screening with the homology-modeled protein structure. Bioorg. Med. Chem. 16 (1), 284-292. doi:10.1016/j.bmc.2007.09.036

Proença, C., Freitas, M., Ribeiro, D., Oliveira, E. F. T., Sousa, J. L. C., Tomé, S. M., et al. (2017). a-Glucosidase inhibition by flavonoids: an in vitro and in silico structure-activity relationship study. J. Enzyme Inhib. Med. Chem. 32 (1), 1216-1228. doi:10.1080/14756366.2017.1368503

Reusch, J. E., and Manson, J. E. (2017). Management of type 2 diabetes in 2017: getting to goal. JAMA 317(10),1015-1016. doi:10.1001/jama.2017.0241

Sun, H., Song, X., Tao, Y., Li, M., Yang, K., Zheng, H., et al. (2018). Synthesis \& a-glucosidase inhibitory \& glucose consumption-promoting activities of flavonoid-coumarin hybrids. Future Med. Chem. 10 (9), 1055-1066. doi:10. 4155/fmc-2017-0293

Tagami, T., Yamashita, K., Okuyama, M., Mori, H., Yao, M., and Kimura, A. (2013). Molecular basis for the recognition of long-chain substrates by plant \& alphaglucosidase. J. Biol. Chem. 288 (26), 19296-19303. doi:10.1074/jbc.M113.465211

Tang, C., Zhu, L., Chen, Y., Qin, R., Mei, Z., Xu, J., et al. (2014). Synthesis and biological evaluation of oleanolic acid derivative-chalcone conjugates as aglucosidase inhibitors. RSC Adv. 4, 10862-10874. doi:10.1039/c3ra46492j

Wang, Y., Ma, L., Pang, C., Huang, M., Huang, Z., and Gu, L. (2004). Synergetic inhibition of genistein and d-glucose on a-glucosidase. Bioorg. Med. Chem. Lett. 14 (11), 2947-2950. doi:10.1016/j.bmcl.2004.03.035

World Health Organization. (2020). Diabetes. Available at: https://www.who.int/ news-room/fact-sheets/detail/diabetes [Accessed August 27, 2020]. 
Ye, G. J., Lan, T., Huang, Z. X., Cheng, X. N., Cai, C. Y., Ding, S. M., et al. (2019). Design and synthesis of novel xanthone-triazole derivatives as potential antidiabetic agents: $\alpha$-Glucosidase inhibition and glucose uptake promotion. Eur. J. Med. Chem. 177, 362-373. doi:10.1016/j.ejmech.2019.05.045

Zeng, Z., Yin, X., Wang, X., Yang, W., Liu, X., and Hong, Y. (2019). Synthesis of water soluble pentacyclic dihydroxyterpene carboxylic acid derivatives coupled amino acids and their inhibition activities on a-glucosidase. Bioorg. Chem. 86, 277-287. doi:10.1016/j.bioorg.2019.02.001

Conflict of Interest: Author JIZ was employed by the company Lunan Pharmaceutical Group Corporation.
The remaining authors declare that the research was conducted in the absence of any commercial or financial relationships that could be construed as a potential conflict of interest.

Copyright $\odot 2021$ Liu, Hao, Bian, Ge, Lu, Xie, Wang, Tao, Yuan, Zhang, Zhang, Jiang and Zhu. This is an open-access article distributed under the terms of the Creative Commons Attribution License (CC BY). The use, distribution or reproduction in other forums is permitted, provided the original author(s) and the copyright owner(s) are credited and that the original publication in this journal is cited, in accordance with accepted academic practice. No use, distribution or reproduction is permitted which does not comply with these terms. 